\title{
Molecular characterization and genetic structure of the Nero Siciliano pig breed
}

\author{
Anna Maria Guastella ${ }^{1}$, Andrea Criscione ${ }^{1}$, Donata Marletta ${ }^{1}$, Antonio Zuccaro ${ }^{1}$, Luigi Chies ${ }^{2}$ \\ and Salvatore Bordonaro ${ }^{1}$ \\ ${ }^{1}$ Dipartimento di Scienze Agronomiche, Agrochimiche e delle Produzioni Animali, \\ Università degli Studi di Catania, Catania, Italy. \\ ${ }^{2}$ Dipartimento di Scienze e Tecnologie Agro-Forestali ed Ambientali, \\ Università degli Studi Mediterranea di Reggio Calabria, Reggio Calabria, Italy.
}

\begin{abstract}
Nero Siciliano is an autochthonous pig breed that is reared mainly in semi-extensive systems in northeastern Sicily. Despite its economic importance and well-appreciated meat products, this breed is currently endangered. Consequently, an analysis of intra-breed variability is a fundamental step in preserving this genetic resource and its breeding system. In this work, we used 25 microsatellite markers to examine the genetic composition of 147 unrelated Nero Siciliano pigs. The total number of alleles detected $(249,9.96$ per locus) and the expected heterozygosity $(0.708)$ indicated that this breed had a high level of genetic variability. Bayesian cluster analysis showed that the most likely number of groups into which the sample could be partitioned was nine. Based on the proportion of each individuals genome derived from ancestry, pigs with at least $70 \%$ of their genome belonging to one cluster were assigned to that cluster. The cluster size ranged from 7 to $17(n=108)$. Genetic variability in this sub-population was slightly lower than in the whole sample, genetic differentiation among clusters was moderate $\left(F_{S T} 0.125\right)$ and the $F_{I S}$ value was 0.011. NeighborNet and correspondence analysis revealed two clusters as the most divergent. Molecular coancestry analysis confirmed the good within-breed variability and highlighted the clusters that retained the highest genetic diversity.
\end{abstract}

Key words: cluster analysis, coancestry, intra-breed genetic variation, microsatellites, Nero Siciliano pig breed.

Received: November 12, 2009; Accepted: May 14, 2010.

\section{Introduction}

In recent decades, many livestock breeds have experience a severe loss of biodiversity that has markedly affected animal production systems, especially in marginal areas. Attempts to reverse this negative trend have led to research in the preservation and exploitation of local animal breeds, with efforts to identify and reintroduce potentially important genetic traits that have been overlooked by globalized production systems. Autochthonous breeds require careful molecular and morphological characterization that takes into account potential influences of the environment in which they were originally developed and adapted.

Nero Siciliano is an ancient black pig breed that originated in Sicily and has always been reared in extensive and semi-extensive systems on this island. Currently, most of the farms that rear autochthonous black pigs (1800 pigs, $70 \%$ of the entire population for this breed) are located mainly in the mountainous area of Nebrodi in northeastern

Send correspondence to Donata Marletta. Dipartimento di Scienze Agronomiche, Agrochimiche e delle Produzioni Animali (DACPA), via Valdisavoia 5, 95125 Catania, Italy. E-mail: d.marletta @ unict.it.
Sicily. Black pigs are rustic animals that thrive on roughage and a limited food supply, in addition to being resistant to diseases in harsh conditions. This breed still retains its distinctiveness thanks to the geographical and orographical characteristics of the island and breeding area, but runs a high risk of losing its original traits because of the lack of a suitable plan to safeguard and exploit its production.

Nero Siciliano pigs grow slowly and yield tasty meat and fat (Pugliese et al., 2003) used to produce high quality meat, including salami and cured ham that are very appreciated by local consumers. The creation of a Protected Designation of Origin (PDO) label for Nero Siciliano meat and other related products has helped to safeguard and preserve this breed, and has led to an important increase $(21 \%)$ in the number of farms and in sow rearing $(24 \%)$ in the last three years. Despite this renewed interest of breeders and consumers, only 850 breeding sows are currently being reared and the Nero Siciliano breed is included in the list of endangered autochthonous breeds.

Genetic characterization is a fundamental prerequisite for managing genetic resources. A recent morphome- 
tric analysis of Nero Siciliano pigs involving 13 body measurements highlighted the low-medium size of this breed when compared with other Italian breeds (Guastella et al., 2009). Genetic variability has also been assessed based on the use of different genetic markers (Russo et al., 2004; D'Alessandro et al., 2007; Davoli et al., 2008). In particular, microsatellite markers have been particularly useful for quantifying genetic variation within and among several European pig breeds, including Nero Siciliano (SanCristobal et al., 2006).

The aim of this study was to use microsatellite markers to assess the genetic variability and genetic structure of Nero Siciliano pigs reared in the Nebrodi mountains, in order to provide suitable data for conservation strategies.

\section{Material and Methods}

\section{Sample collection and microsatellite analysis}

A representative sample of 147 Nero Siciliano pigs (22 boars and 125 sows) was selected from 22 farms in 11 communes (Alcara Li Fusi, Brolo, Capizzi, Caronia, Floresta, Longi, Mirto, Sanfratello, San Salvatore di Fitalia, Raccuja and Tortorici) in the Nebrodi area; first- and second-degree relatives were avoided. The Nebrodi area is part

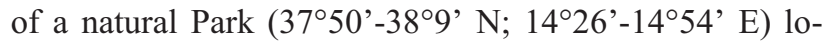
cated $100-1700 \mathrm{~m}$ above sea level, and is where the Nero Siciliano breed originated and is still extensively bred. The sample size ranged from 2 to 15 pigs per farm, depending on the herd size. Only pigs that met the morphological standard for the breed were sampled, and digital photographs of each animal were taken. Farmers were also asked about the breeding strategies that they employed to select animals with an exclusively native Sicilian germplasm: farms in which there had been recent crossbreeding with commercial breeds were excluded from the sampling.

For each pig, $10 \mathrm{~mL}$ of peripheral blood was collected in K3-EDTA tubes. DNA was extracted from blood using the commercial Illustra blood genomic Prep Mini Spin kits (GE Healthcare, Little Chalfont, UK). Genetic characterization was done with a set of 25 microsatellite markers (Table S1) chosen from a list maintained by the Pig Biodiversity project and USDA MARC database. The markers were chosen based on preliminary data about their degree of heterozygosity and polymorphism obtained from small samples of Nero Siciliano and other local Italian pig populations. Six PCR multiplex reactions and two single PCR reactions were done to amplify the microsatellites according to standard protocols. The amplicons $(2 \mu \mathrm{L})$ were mixed with $4 \mu \mathrm{L}$ of loading buffer containing formamide and 350 TAMRA as an internal size standard (Applied Biosystems, Warrington, UK). Individual genotypes were determined with an ABI PRISM ${ }^{\circledR} 377$ sequencer equipped with Genescan Analysis ${ }^{\circledR}$ v.3.1.2 and Genotyper ${ }^{\circledR}$ v. 2.5 softwares (Applied Biosystems, Foster City, CA, USA).

\section{Statistical analysis}

Individual multilocus genotypes were analyzed by Molkin v. 3.0 software (Gutierrez et al., 2005) to calculate the main parameters of genetic variability. For each locus and group of pigs, the allele frequencies, private alleles $\left(A_{p}\right)$, effective number of alleles $\left(A_{e}\right)$, and observed $\left(H_{o}\right)$ and expected $\left(\mathrm{H}_{\mathrm{e}}\right)$ heterozygosities were calculated. Molecular coancestry coefficients and kinship distances $\left(D_{k}\right)$ weighted by polymorphic information content (PIC) were also assessed. In addition, the contribution of the different groups of pigs to the overall or total diversity was also inferred, according to Caballero and Toro (2002) and Petit et al. (1998).

Genepop v. 4.0 (Rousset, 2007) was used to perform the score test for Hardy-Weinberg equilibrium (Rousset and Raymond, 1995) per locus using a Markov chain algorithm implemented with 10,000 dememorizations, 200 batches and 5000 iterations per batch. The presence of null alleles was tested with MICRO-CHECKER v. 2.2.3 (Van Oosterhout et al., 2004), using Bonferroni adjustments.

FSTAT v.2.9.3 software (Goudet, 2001) was used to estimate the $F_{\mathrm{IT}}, F_{\mathrm{ST}}$ and $F_{\mathrm{IS}}$ statistics (Weir and Cockerham, 1984) and their significance was inferred by methods based on randomisation. Multiple tests of significance were corrected by the sequential Bonferroni method (Rice, 1989).

The model-based approach proposed by Falush et al. (2003) in the software STRUCTURE v.2.2 was used to assess the genomic clustering of the sample. Individual pigs were probabilistically assigned to two or more subpopulations on the basis of their multilocus genotype, assuming that they were admixed. As suggested by the authors, the admixture model associated with the option of correlated allele frequencies was used to infer the population structure. The run length was set to 100,000 burn-ins followed by 100,000 iterations. This setting produced consistent estimations that were not significantly altered by a longer burn-in or Markov chain Monte Carlo (MCMC). The range of possible clusters $(K)$ tested was from 1 to 15 , and 10 runs were done for each $K$. CLUMPP software (Jakobsson and Rosenberg, 2007) was subsequently used to find the optimal alignment of the 10 replicate cluster analyses of the same $K$. The similarity coefficient G', which is also a measure of the constancy over runs, was used to define the population structure. The mean membership matrix across replicates was plotted with the program DISTRUCT (Rosenberg, 2004). The method reported by Evanno et al. (2005) was also used to estimate the most likely number of $K$ that explained the sample structure.

Reynolds' pairwise distances (Reynolds et al., 1983), used to assess the genetic relationship between clusters, were calculated with the software Phylip v. 3.67 (Felsenstein, 2005). A multivariate method of correspondence analysis allowed the simultaneous representation of inferred clusters. GENETIX v.4.05 software (Belkhir et al., 
2004) was used to spatially plot clusters and individuals based on the allele frequencies of all loci and a correspondence analysis in which the Chi-square distances served to judge the proximity of the clusters.

\section{Results}

Nero Siciliano pigs showed high genetic variability (Table 1). Two hundred and forty-nine alleles were detected, with 5 (locus S0026) to 19 (locus S0005) alleles per locus and an average number per locus (9.96) that was fairly high. The effective number of alleles $\left(\mathrm{A}_{e}\right)$, which takes into account the expected heterozygosity $\left(\mathrm{H}_{\mathrm{e}}\right)$, showed that $\mathrm{S} 0005$ was the most polymorphic locus and SW951 the least polymorphic. The expected heterozygosity was higher than that observed at each locus and ranged from 0.245 to 0.907 (mean: 0.708 ). The estimated polymorphic information content (PIC) ranged from 0.236 at SW951 to 0.901 at $S 0005$ (data not shown). Overall, Nero

Table 1 - Number of observed (NA) and effective $\left(\mathrm{A}_{\mathrm{e}}\right)$ alleles, observed $\left(\mathrm{H}_{\mathrm{o}}\right)$ and expected heterozygosity $\left(\mathrm{H}_{\mathrm{e}}\right)$, and $\mathrm{F}_{\mathrm{IS}}$ per locus.

\begin{tabular}{|c|c|c|c|c|c|}
\hline Locus & NA & $A_{e}$ & $\mathrm{H}_{\mathrm{o}}$ & $\mathrm{H}_{\mathrm{e}}$ & $\mathrm{F}_{\text {IS }}$ \\
\hline$S W 240$ & 16 & 5.66 & 0.738 & 0.823 & $0.107^{*}$ \\
\hline SW857 & 9 & 5.24 & 0.762 & $0.809 * * *$ & 0.061 \\
\hline S0155 & 7 & 3.25 & 0.632 & 0.692 & 0.091 \\
\hline S0101 & 8 & 3.82 & 0.732 & 0.738 & 0.012 \\
\hline S0005 & 19 & 10.78 & 0.782 & $0.907 * *$ & $0.141 * * *$ \\
\hline S0355 & 8 & 1.94 & 0.429 & 0.484 & 0.117 \\
\hline SW936 & 11 & 3.85 & 0.676 & 0.740 & 0.091 \\
\hline$S W 72$ & 9 & 4.02 & 0.731 & 0.751 & 0.030 \\
\hline SW911 & 7 & 3.97 & 0.600 & $0.748^{* * *}$ & $0.201 * *$ \\
\hline S0228 & 7 & 1.68 & 0.380 & 0.403 & 0.060 \\
\hline SW1928 & 8 & 3.65 & 0.695 & 0.726 & 0.046 \\
\hline SW1873 & 13 & 6.73 & 0.667 & $0.851 * *$ & $0.221 * * *$ \\
\hline SW1695 & 14 & 8.75 & 0.810 & $0.886^{*}$ & 0.089 \\
\hline SW1556 & 6 & 2.08 & 0.293 & $0.519 * * *$ & $0.439 * * *$ \\
\hline$S W 1370$ & 14 & 6.14 & 0.738 & $0.837 *$ & $0.122 *$ \\
\hline SW1035 & 12 & 2.37 & 0.500 & $0.578^{*}$ & $0.138^{*}$ \\
\hline SWR153 & 8 & 3.20 & 0.600 & 0.688 & 0.131 \\
\hline SW2038 & 11 & 7.14 & 0.760 & 0.860 & $0.119^{* *}$ \\
\hline S0017 & 10 & 2.62 & 0.558 & 0.618 & 0.101 \\
\hline SW1823 & 12 & 7.53 & 0.807 & 0.867 & 0.073 \\
\hline S0026 & 5 & 2.29 & 0.521 & 0.562 & 0.077 \\
\hline$S W 24$ & 11 & 5.45 & 0.782 & 0.817 & 0.045 \\
\hline SW951 & 6 & 1.32 & 0.221 & $0.245^{*}$ & 0.102 \\
\hline SW632 & 12 & 6.27 & 0.828 & 0.840 & 0.019 \\
\hline$S 0090$ & 6 & 3.51 & 0.603 & $0.715^{*}$ & $0.160 * *$ \\
\hline Average & 9.96 & 4.53 & 0.634 & $0.708 * * *$ & $0.109 * * *$ \\
\hline
\end{tabular}

Deviations from $\mathrm{H}-\mathrm{W}$ equilibrium and significance of the $\mathrm{F}_{\mathrm{IS}}$ values are indicated by asterisks $\left(* \mathrm{p}<0.05,{ }^{* *} \mathrm{p}<0.01\right.$ and $\left.{ }^{* * *} \mathrm{p}<0.001\right)$.
Siciliano pigs showed Hardy-Weinberg disequilibrium, with significant deviations from equilibrium being observed for 10 microsatellites (Table 1). The presence of null alleles, inferred for five loci (S005, SW911, SW1873, SW1556, SW2038), could explain the excess of homozygotes and the deviation from genetic equilibrium at these loci. The heterozygote deficiency within populations $\left(F_{\mathrm{IS}}\right)$ was significant for nine loci. The overall $F_{\text {IS }}$ coefficient for the loci was 0.109 , indicating a significant $(\mathrm{p}<0.001)$ excess of homozygotes in the whole sample.

The level of population structuring was quite high. Model-based clustering of the microsatellite genotypes revealed that the likelihood variance of the observed data initially increased by the predefined number of clusters to reach a peak value at $K=9$ and then decreased. As indicated by Pritchard et al. (2000), nine is the smallest value of $K$ that captures the major structure in the dataset. Based on the method proposed by Evanno et al. (2005), two clear peaks at $K=9$ and $K=2$ (the latter being particularly indicative of a very low likelihood variance) were observed in the $\Delta K$ distribution. The highest values of the similarity coefficient G' (>95\%) were detected at $K=2, K=7$ and $K=9$. Based on these three approaches, we assumed $K=9$ to be the most likely number of clusters.

Based on the average matrix of membership (data not shown), animals with at least $70 \%$ of their genome belonging to one cluster were assigned to that cluster. The cluster size ranged from 7 to 17 (for a subsample of 108 of the original 147 pigs). Table 2 shows the genetic parameters of diversity for the nine clusters and the subsample of 108 pigs. The average genetic differentiation among inferred clusters was moderate $\left(F_{\mathrm{ST}} 0.125 ; \mathrm{p}=0.001\right)$. The genetic variability of this subsample was still high with the overall $F_{\text {IS }}$ value (0.011) not significantly different from zero, indicating frequent random mating. The observed and expected heterozygosities were slightly lower than in the whole sample of 147 pigs.

Among the 22 farms sampled, nine were highly homogenous, with $>70 \%$ of the genome belonging to a cluster. In contrast, animals from three farms that were particularly active in fattening pigs for meat production and pork products showed a wide genome distribution among clusters. Some clusters $(K 7, K 5$ and $K 3)$ consisted of individuals from single farms, whereas two (K9 and $K 4)$ consisted of pigs from different herds reared in the same area.

The rarefacted number of alleles (Ar), which measures the contribution of alleles weighted by the sample size, ranged from $3.01(K 7)$ to $4.40(K 1)$. Thirty-three private alleles were detected in the model-inferred group of pigs. Each cluster was characterized by at least one unique allele: high frequencies were observed for alleles $S W 1695$ (167 bp), SW1928 (85 bp) and S0005 (258 bp) in K3 (0.39), $K 7$ (0.36) and $K 2(0.25)$, respectively.

NeighborNet built on Reynolds' distances revealed a close linkage among most of the clusters: $K 7$ and $K 2$ were 
Table 2 - Number of heads, average number of observed (NA) alleles per locus, private alleles $\left(\mathrm{A}_{\mathrm{p}}\right)$ with the highest allele frequency in parentheses, observed $\left(\mathrm{H}_{\mathrm{o}}\right)$ and expected $\left(\mathrm{H}_{\mathrm{e}}\right)$ heterozygosities, and $\mathrm{F}_{\mathrm{IS}}$ values per cluster and for the subsample of 108 pigs. The contribution of allelic richness $(\mathrm{CAr})$ and gene diversity (CGD) per cluster to the diversity of the subsample is also shown.

\begin{tabular}{lccccccccc}
\hline Cluster & Heads & $\mathrm{NA}$ & $\mathrm{A}_{\mathrm{p}}$ & $\mathrm{H}_{\mathrm{o}}$ & $\mathrm{H}_{\mathrm{e}}$ & $\mathrm{F}_{\mathrm{IS}}$ & CAr & CGD \\
\hline $\mathrm{K} 1$ & 13 & 6.32 & $4(11.5)$ & 0.675 & 0.669 & 0.033 & 2.059 & -1.044 \\
$\mathrm{~K} 2$ & 8 & 3.88 & $5(25.0)$ & 0.670 & 0.600 & -0.046 & 1.154 & -1.136 \\
$\mathrm{~K} 3$ & 10 & 4.48 & $5(38.9)$ & 0.574 & $0.621^{* *}$ & $0.137^{*}$ & 1.048 & -0.498 \\
$\mathrm{~K} 4$ & 12 & 4.88 & $2(16.7)$ & 0.568 & $0.601^{*}$ & $0.106^{*}$ & 0.798 & -0.082 \\
$\mathrm{~K} 5$ & 12 & 4.16 & $1(8.3)$ & 0.627 & 0.556 & -0.084 & -0.724 & 0.319 \\
$\mathrm{~K} 6$ & 15 & 5.72 & $4(13.3)$ & 0.683 & 0.642 & -0.029 & 0.814 & -0.354 \\
$\mathrm{~K} 7$ & 14 & 3.72 & $4(35.7)$ & 0.543 & 0.511 & -0.026 & -1.535 & 0.332 \\
$\mathrm{~K} 8$ & 17 & 5.76 & $4(11.8)$ & 0.629 & 0.616 & 0.002 & -0.365 & 0.747 \\
$\mathrm{~K} 9$ & 7 & 4.12 & $4(14.3)$ & 0.632 & 0.607 & 0.032 & 0.456 & -0.278 \\
Total & 108 & 9.52 & 33 & 0.622 & 0.706 & 0.011 & - & - \\
\hline
\end{tabular}

the most divergent in terms of average distance, with values of 0.190 and 0.182 , respectively. The lowest pairwise distance was between $K 6$ and $K 8$, and the highest between $K 7$ and $K 5$ (data not shown).

Correspondence analysis provided an alternative spatial representation of individuals and clusters scattered in the metric space (Figure 1). The first two axes contributed almost equally to the total inertia $(16.84 \%$ and $15.96 \%$, respectively). With regard to the dispersion in the first and second axes, S0017 (155 bp), SW1928 (85 bp), SW240 (122 bp) and $S 0017$ (155 bp) were the most important alleles, each with a contribution $>5.7 \%$.

The spatial dispersion of clusters and related individuals was determined mainly by four alleles that had very different frequencies among clusters, and by high frequency alleles detected exclusively in a given cluster. In particular, allele $S W 1928$ ( $85 \mathrm{bp}$ ), which was found only in $K 7$ at a high frequency (35.7\%), contributed significantly to dispersion in the first and second axes $(3.8 \%$ and $2.4 \%$, respectively); an additional three alleles contributed more than $5 \%$ to dispersion in the first two axes and occurred at very high frequencies, i.e., $50 \%$ for $S W 240$ (122 bp) in K2, $56 \%$ for $S 0017$ (155 bp) in $K 2$ and $82 \%$ for $S W 1873$ (136 bp) in K7.

Molecular coancestry in each cluster ranged from 0.287 in $K 1$ to 0.389 in $K 7$. Self-coancestry and the inbreeding coefficient showed the same trend: the lowest values were detected in $K 6$, the highest in $K 3$. Kinship distances ranged from 0.277 to 0.361 . The contribution to the diversity of the whole sample is shown in Table 2. Cluster 2 contributed the most to the total genetic diversity (CGD) and $K 8$ the least, when assessed according to Caballero and

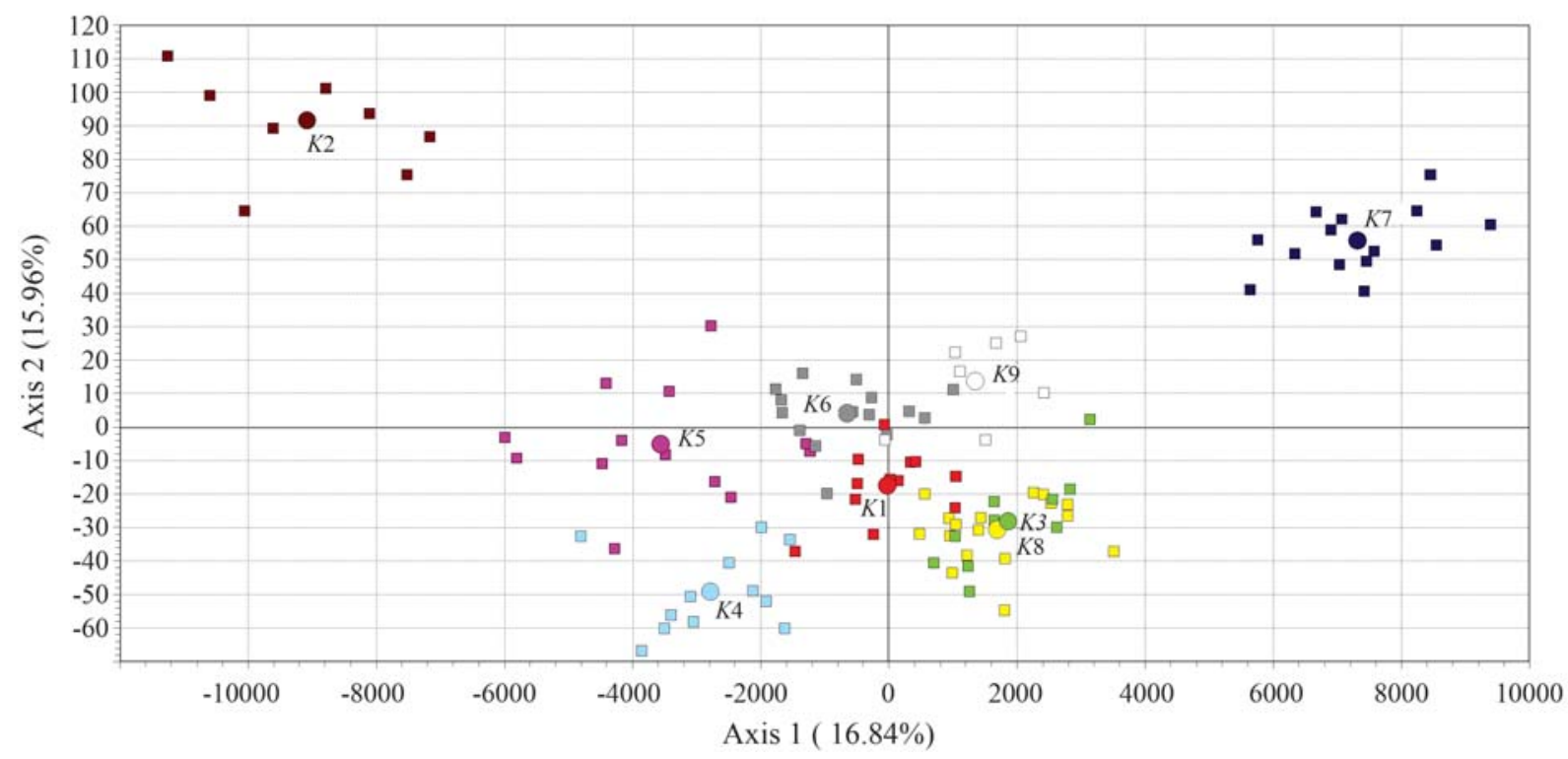

Figure 1 - Summary of individual and cluster positions according to the first and second axis of dispersion in correspondence analysis. Circles and squares indicate clusters and individuals, respectively. 
Toro (2002). However, when the rarefacted number of alleles was considered, clusters $K 1$ and $K 7$ provided the highest and lowest contributions (CAr), respectively (Petit et al., 1998).

\section{Discussion}

Nero Siciliano pigs showed considerable genetic variability and diversity, expressed in terms of the number of alleles per locus (NA) and expected heterozygosity $\left(\mathrm{H}_{\mathrm{e}}\right)$ : the values for these parameters were higher than for several European breeds (NA $=4.5$ on average, $\left.\mathrm{H}_{\mathrm{e}}=0.43-0.68\right)$, including Iberian breeds $\left(\mathrm{NA}=3.44-5.86, \mathrm{H}_{\mathrm{e}}=0.46-0.64\right)$ (Martinez et al., 2000), seven native and commercial breeds reared in Portugal (NA $\left.=3.77-6.27, \mathrm{H}_{\mathrm{e}}=0.49-0.69\right)$ (Vicente et al., 2008), and a population of Nero Siciliano pigs $\left(\mathrm{NA}=6.70, \mathrm{H}_{\mathrm{e}}=0.67\right)$ studied by SanCristobal et al. (2006). These values were also greater than those reported for Korean native pigs (NA $\left.=3.44, \mathrm{H}_{\mathrm{e}}=0.49\right)$ (Kim et al., 2005), Cuban Criollo pigs (NA $\left.=8.2, \mathrm{H}_{\mathrm{e}}=0.65\right)$ (Martinez et al., 2005) and most Chinese breeds (NA $=6.25-11.55$, $\left.\mathrm{H}_{\mathrm{e}}=0.57-0.77\right)$ (Li et al., 2004).

The number of effective alleles (4.53) was higher than in European breeds (2.74 on average) and Nero Siciliano pigs (4.03) studied by SanCristobal et al. (2006) and higher than or comparable to those reported for several Chinese breeds (1.69-5.62) (Li et al., 2004; Fang et al., 2005), except for Lingao (4.76; Fang et al., 2005) and Nang Yang Black, Sheng Xian Spotted and Hai Nan (5.12, 5.21 and 5.62, respectively) (Li et al., 2004). Conversely, the number of effective alleles and expected heterozygosity in Nero Siciliano pigs was lower than in the Indian Ankamali breed $\left(\mathrm{Ae}=5.34, \mathrm{H}_{\mathrm{e}}=0.83\right)($ Behl et al., 2006).

The significant deviation from Hardy-Weinberg equilibrium observed at 10 loci may reflect the presence of null alleles at some loci and the Wahlund effect, i.e., a reduction in the observed heterozygosity that occurs in subdivided populations. Genetic substructures in Nero Siciliano pigs apparently resulted from different management strategies across herds and inbreeding within herds. In the Nebrodi area, farmers use their own boars for a year or more, with only a limited exchange of animals among farms and herds. The within-group inbreeding values $\left(F_{\mathrm{IS}}\right)$ support this hypothesis (Table 2).

The unsupervised analysis of population structure detected nine homogenous groups in the sample analyzed and determined the corresponding fraction of the individual's genome derived from ancestry in each cluster. Cluster $K 1$ showed the highest variability in the number of alleles and heterozygosity, whereas $K 7$ showed the least. These results agreed with the values for molecular coancestry and were confirmed by analysis of the contribution to the total genetic diversity estimated from the allelic richness.

The negative $F_{\text {IS }}$ values detected in clusters $K 2$ and $K 5$, which represent two closed-cycle farms, probably reflected the proper selection of breed animals among the farms sampled. In these farms, which sell piglets of different ages for production or reproduction, the systematic exchange of genetic resources that occurs between breeding and processing herds probably helps to maintain a high level of diversity. Molecular data confirmed that these two farms had good mating management programs that ensured high genetic variability.

Correspondence analysis provided a more interesting and informative spatial representation of the relationships among the nine clusters with respect to the network built on genetic distances. Clusters $K 2$ and $K 7$ represented the two most distinct group of pigs and accounted for $14.2 \%$ and $12.7 \%$ of the inertia, respectively. Cluster 7 represented a single herd. The farm in question is interesting because it is the only one in the Nebrodi area in which the recessive mutation in the RYR1 (Ryanodine Receptor 1) locus responsible for the Porcine Stress Syndrome (PSS) has been reported (Matassino et al., 2007); ten of the 14 pigs in this cluster were carriers of this recessive allele. Despite their morphological traits and recent successful breeding history, these pigs retain the traces of a possible accidental introgression of other commercial breeds. In addition, the low values for effective alleles and expected heterozygosity in $K 7$ may reflect mating between closely related or inbred animals. Cluster 2 also represented a single farm that was among the first to rationally manage the short chain cycle of breeding-processing-resale. The spatial distribution of $K 2$ pigs and the high contribution of this cluster to overall diversity support the hypothesis that the present herd was derived from different genetic lineages that were incorporated to control the level of inbreeding.

Mitochondrial DNA haplotype analysis has shown that modern European pig breeds belong to a general European cluster (Larson et al., 2005). The populations are often heavily structured and more than half of the European pig diversity can be assigned to local breeds (Ollivier et al., 2005). The results described here indicate a high degree of genetic variability in Nero Siciliano pigs, as previously reported (SanCristobal et al., 2006; Davoli et al., 2007), despite the limited population size of this breed. This is a rather frequent condition for populations reared in extensive systems where there is no systematic selection and planned mating. Part of the observed heterogeneity may have originated from accidental crosses with wild boars (Ollivier et al., 2005) and limited introgression with other Italian breeds (Neapolitan and Casertana), as well as with Iberian breeds and commercial pigs (Russo et al., 2004).

As shown here, the genetic profile established by using neutral markers and the analysis of intra-population structure provided a general outline of the breeding systems applied to Nero Siciliano pigs in the Nebrodi area. The model-based approach identified homogenous groups in the sample, some of which coincided with specific farms and others with breeding areas. Our results indicate that molecular data can be helpful in the selection of parental 
stocks and planned matings as part of strategies to preserve and restore the rational breeding of black pigs. Boars belonging to cluster $K 7$ need to be screened for the PSS syndrome gene and possibly excluded from selection programs in order to eliminate the PSS mutated gene and related PSE (pale, soft, exudative) defect from the population gene pool. Individuals belonging to different clusters could be used in planned matings to maintain a good level of genetic variability and rusticity (stress-resistance) and avoid excessive inbreeding. On the other hand, pigs sharing the same clusters and chosen based on their individual multilocus genotypes may be used in planned matings to preserve the most typical traits in this autochthonous population. Nero Siciliano pigs belonging to the most divergent cluster $(K 2)$ and pigs not included in the most homogenous subpopulation of 108 animals need to be incorporated into selection schemes in order to counterbalance any increase in inbreeding. Such approaches should help to preserve the Nero Siciliano breed and ensure the production of high quality products for local and national markets.

\section{Acknowledgments}

The authors thank Drs. Vincenzo Di Marco, Vincenzo Pruiti and Giuseppe Spartà for technical assistance. This work was funded by Regione Sicilia Assessorato Agricoltura e Foreste Progetto "SUNEBRO".

\section{References}

Behl R, Sheoran N, Behl J and Vijh RK (2006) Genetic analysis of Ankamali pigs of India using microsatellite markers and their comparison with other domesticated Indian pig types. J Anim Breed Genet 123:131-135.

Belkhir K, Borsa P, Chikhi L, Raufaste N and Bonhomme F (2004) GENETIX 4.05, Logiciel sous Windows TM pour la Génétique des Populations. Laboratoire Génome, Populations, Interactions, CNRS UMR 5171, Université de Montpellier II, Montpellier.

Caballero A and Toro MA (2002) Analysis of genetic diversity for the management of conserved subdivided populations. Conserv Genet 3:289-299.

D’Alessandro E, Fontanesi L, Liotta L, Davoli R, Chiofalo V and Russo V (2007) Analysis of the MC1R gene in the Nero Siciliano pig breed and usefulness of this locus for breed traceability. Vet Res Commun 31 (Suppl. 1):389-392.

Davoli R, Zambonelli P, San-Cristobal M, Scotti E, Fontanesi L, Colombo M, Dall'Olio S, Braglia S and Russo V (2007) SNPs and microsatellite markers for genetic diversity study in Italian pig breeds. In: Nanni Costa L, Zambonelli P and Russo V (eds) Proceedings of the $6^{\text {th }}$ International Symposium on the Mediterranean Pig. Università di Bologna, Capo d'Orlando, pp 46-53.

Evanno G, Regnaut S and Goudet J (2005) Detecting the number of clusters of individuals using the software STRUCTURE: A simulation study. Mol Ecol 14:2611-2620.

Falush D, Stephens M and Pritchard JK (2003) Inference of population structure: Extensions to linked loci and correlated allele frequencies. Genetics 164:1567-1587.
Fang M, Hu X, Jiang T, Braunschweig M, Hu L, Du Z, Feng J, Zhang Q, Wu C and Li N (2005) The phylogeny of Chinese indigenous pig breeds inferred from microsatellite markers. Anim Genet 36:7-13.

Guastella AM, Bordonaro S, Zuccaro A, Marletta D, Criscione A and D'Urso G (2009) Morphological and genetic characterization of Nero Siciliano pig population reared in the Nebrodi area. Ital J Anim Sci 8 (Suppl. 2):209.

Gutiérrez JP, Royo LJ, Álvarez I and Goyache F (2005) MolKin v. 2.0: A computer program for genetic analysis of populations using molecular coancestry information. J Hered 96:718721.

Jakobsson M and Rosenberg NA (2007) CLUMPP: A cluster matching and permutation program for dealing with label switching and multimodality in analysis of population structure. Bioinformatics 23:1801-1806.

Kim TH, Kim KS, Choi BH, Yoon DH, Jang GW, Lee KT, Chung HY, Lee HY, Park HY and Lee JW (2005) Genetic structure of pig breeds from Korea and China using microsatellite loci analysis. J Anim Sci 83:2255-2263.

Larson G, Dobney K, Albarella U, Fang M, Matisoo-Smith E, Robins J, Lowden S, Finlayson H, Brand T, Willerslev E, et al. (2005) Worldwide phylogeography of wild boar reveals multiple centers of pig domestication. Science 307:16181621.

Li SJ, Yang SH, Zhao SH, Fan B, Yu M, Wang HS, Li MH, Liu B, Xiong TA and Li K (2004) Genetic diversity analyses of 10 indigenous Chinese pig populations based on 20 microsatellites. J Anim Sci 82:368-374.

Martinez AM, Delgado JV, Rodero A and Vega-Pla JL (2000) Genetic structure of the Iberian pig breed using microsatellites. Anim Genet 31:295-301.

Martinez AM, Pèrez-Pineda E, Vega-Pla JL, Barba C, Velàzquez FJ and Delgado JV (2005) Genetic characterisation of the Cuban Creole pig with microsatellites. Arch Zootec 54:369-375.

Matassino D, Bordonaro S, Castellano N, Guastella AM, Incoronato C, Monaco F, Occidente M, Pane F and Barone CMA (2007) CRC locus screening in some Italian pig ancient autochthonous genetic types (AAGTs). Preliminary results. In: Nanni Costa L, Zambonelli P and Russo V (eds) Proceedings of the $6^{\text {th }}$ International Symposium on the Mediterranean Pig. Università di Bologna, Capo d'Orlando, pp 68-72.

Ollivier L, Alderson L, Gandini GC, Foulley JL, Haley CS, Joosten R, Rattink AP, Harlizius B, Groenen MAM, Amigues $Y$ et al. (2005) An assessment of European pig diversity using molecular markers: Partitioning of diversity among breeds. Conserv Genet 6:729-741.

Petit RJ, El Mousadik A and Pons O (1998) Identifying populations for conservation on the basis of genetic markers. Conserv Biol 12:844-855.

Pritchard JK, Stephens M and Donnely P (2000) Inference of population structure using multilocus genotype data. Genetics 155:945-949.

Pugliese C, Madonna G, Chiofalo V, Margotta S, Acciaioli A and Gandini G (2003) Comparison of the performances of Nero Siciliano pigs reared indoors and outdoors. 1. Growth and carcass composition. Meat Sci 65:825-831. 
Reynolds J, Weir BS and Cockerham C (1983) Estimation of the coancestry coefficient: Basis for a short-term genetic distance. Genetics 105:767-779.

Rice WR (1989) Analysing tables of statistical tests. Evolution 43:223-225.

Rosenberg NA (2004) Distruct: A program for the graphical display of population structure. Mol Ecol Notes 4:137-138.

Rousset F (2007) Genepop'007: A complete re-implementation of the GENEPOP software for Windows and Linux. Mol Ecol Notes 8:103-106.

Rousset F and Raymond M (1995) Testing heterozygote excess and deficiency. Genetics 140:1413-1419.

Russo V, Fontanesi L, Davoli R, Chiofalo L, Liotta L and Zumbo A (2004) Analysis of single nucleotide polymorphisms in major and candidate genes for production traits in Nero Siciliano pig breed. Ital J Anim Sci 3:19-29.

SanCristobal M, Chevalet C, Haley CS, Joosten R, Rattink AP, Harlizius B, Groenen MA, Amigues Y, Boscher MY, Russell G et al. (2006) Genetic diversity within and between European pig breeds using microsatellite markers. Anim Genet 37:189-198.

Van Oosterhout C, Hutchinson WF, Wills DPM and Shipley P (2004) MICRO-CHECKER: Software for identifying and correcting genotyping errors in microsatellite data. Mol Ecol Notes 4:535-538.

Vicente AA, Carolino MI, Sousa MCO, Ginja C, Silva FS, Martinez AM, Vega-Pla JL, Carolino N and Gama LT (2008) Genetic diversity in native and commercial breeds of pigs in Portugal assessed by microsatellites. J Anim Sci 86:24962507.
Weir BS and Cockerham CC (1984) Estimating F-statistics for the analysis of population structure. Evolution 38:1358-1370.

\section{Internet Resources}

Felsenstein J (2005) PHYLIP (Phylogeny Inference Package), v. 3.6. Distributed by the author. Department of Genome Sciences, University of Washington, Seattle. Available from http://evolution.genetics.washington.edu/phylip/getme.html (December 3, 2009).

Goudet J (2001) FSTAT, a program to estimate and test gene diversities and fixation indices (version 2.9.3). Available from http://www.unil.ch/izea/softwares/fstat.html (December 3, 2009).

Pig Biodiversity Project, http://www.projects.roslin.ac.uk/ pigbiodiv/markers.html (December 3, 2009).

USDA MARC database, http://www.genome.iastate.edu/ pigs/maps/marc.html (December 3, 2009).

\section{Supplementary Material}

The following material is available online for this article:

Table S1 - Multiple (M) and single (S) PCR, locus name and chromosomal location, and primer sequences and dyes used in PCR

This material is available as part of the online article from http://www.scielo.br/gmb.

Associate Editor: Bertram Brenig

License information: This is an open-access article distributed under the terms of the Creative Commons Attribution License, which permits unrestricted use, distribution, and reproduction in any medium, provided the original work is properly cited. 
Table S1 - Multiple (M) and single (S) PCR, locus name and chromosomal location, and primer sequences and dyes used in PCR.

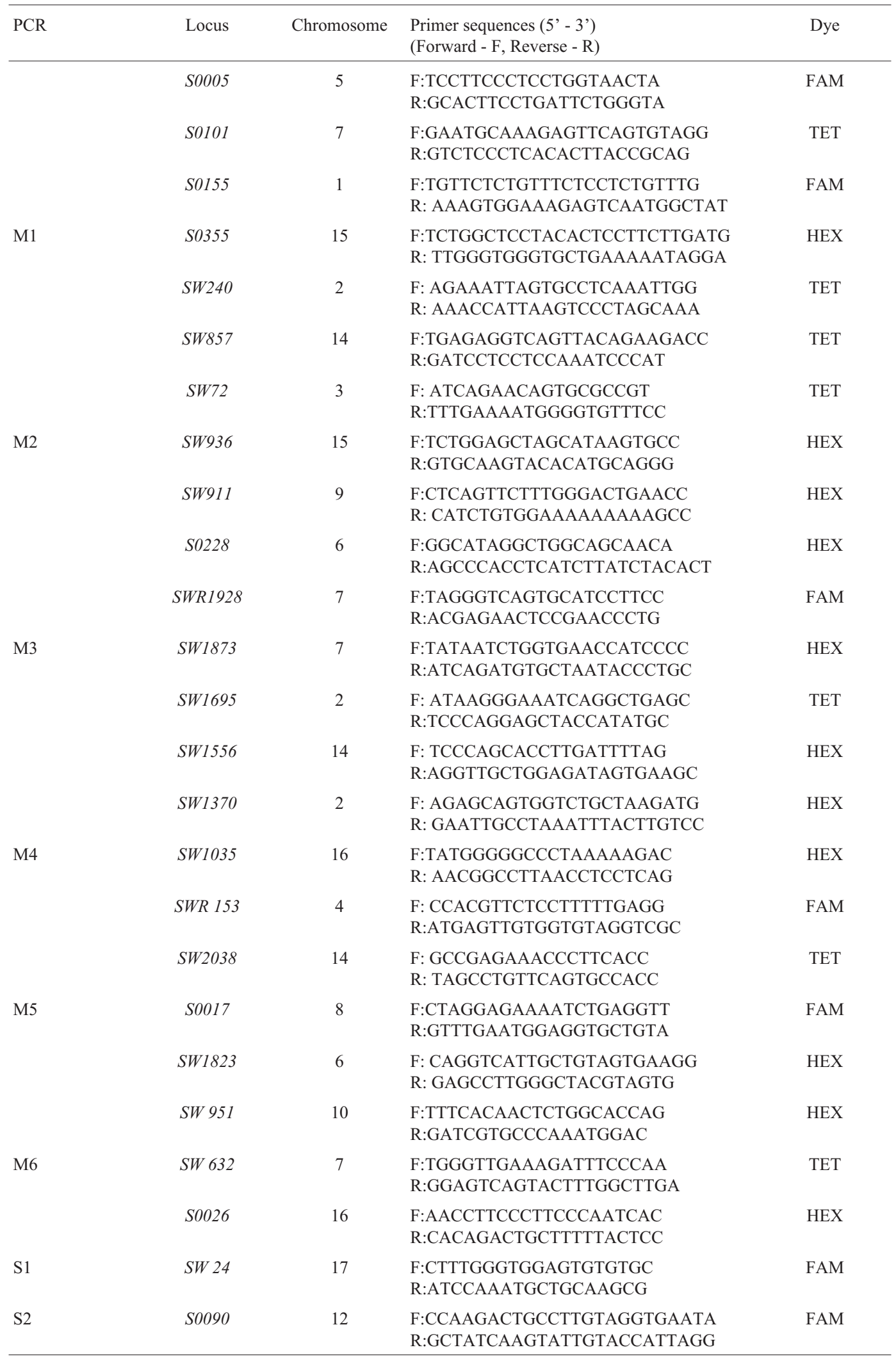

FAM - blue, HEX - green, TET - yellow. 